\title{
CRITICAL TEST OF SIMULATIONS OF CHARGE-EXCHANGE-INDUCED X-RAY EMISSION IN THE SOLAR SYSTEM
}

\author{
R. Ali, ${ }^{1}$ P. A. Neill, ${ }^{2}$ P. Beiersdorfer, ${ }^{3}$ C. L. Harris, ${ }^{4}$ D. R. Schultz, ${ }^{5}$ and P. C. Stancil ${ }^{6}$
}

\begin{abstract}
Experimental and theoretical state-selective X-ray spectra resulting from single-electron capture in charge exchange $(\mathrm{CX})$ collisions of $\mathrm{Ne}^{10+}$ with $\mathrm{He}, \mathrm{Ne}$, and $\mathrm{Ar}$ are presented for a collision velocity of $933 \mathrm{~km} \mathrm{~s}^{-1}\left(4.54 \mathrm{keV}\right.$ nucleon $\left.{ }^{-1}\right)$, comparable to the highest velocity components of the fast solar wind. The experimental spectra were obtained by detecting scattered projectiles, target recoil ions, and X-rays in coincidence; with simultaneous determination of the recoil ion momenta. Use and interpretation of these spectra are free from the complications of non-coincident total X-ray measurements that do not differentiate between the primary reaction channels. The spectra offer the opportunity to test critically the ability of CX theories to describe such interactions at the quantum orbital angular momentum level of the final projectile ion. To this end, new classical trajectory Monte Carlo calculations are compared here with the measurements. The current work demonstrates that modeling of cometary, heliospheric, planetary, and laboratory X-ray emission based on approximate state-selective CX models may result in erroneous conclusions and deductions of relevant parameters.
\end{abstract}

Subject headings: atomic data - atomic processes - comets: general — solar wind-X-rays: general

\section{Introduction}

X-ray and extreme ultraviolet (EUV) emission has been detected from more than 20 comets since the first observation by Lisse et al. (1996). The charge exchange (CX) mechanism between highly charged solar wind (SW) minor heavy ions and cometary neutrals suggested by Cravens (1997) is now recognized as the primary process responsible for the observed emission (see,

\footnotetext{
${ }^{1}$ Department of Physics, The University of Jordan, Amman 11942, Jordan; ramimali@ju.edu.jo

${ }^{2}$ Department of Physics, University of Nevada, Reno, NV 89557, USA; paul@physics.unr.edu

${ }^{3}$ Lawrence Livermore National Laboratory, 7000 East Avenue, L-260, Livermore, CA 94550, USA; beiersdorfer@llnl.gov

${ }^{4}$ Natural Sciences Division, Gulf Coast Community College, Panama City, FL 32401, USA; charris@ gulfcoast.edu

${ }^{5}$ Physics Division, Oak Ridge National Laboratory, Oak Ridge, TN 37831, USA; schultzd@ornl.gov

${ }^{6}$ Department of Physics and Astronomy, University of Georgia, Athens, GA 30602, USA; stancil@physast.uga.edu
}

e.g., Lisse et al. 2001; Krasnopolskv \& Mumma 2001; Krasnopolskv et al. 2002; Beiersdorfer et al. 2003; Kharchenko et al. 2003; Willingale et al. 2006; Bodewits et al. 2007; Lisse et al. 2007, and references therein). In the SWCX mechanism, electrons are captured from cometary neutrals by SW ions into excited states of the product ions, which may then decay radiatively and in the process emit X-ray radiation. The SWCX mechanism has been invoked with various degrees of sophistication to model and interpret cometary X-ray and EUV emission spectra (Häberli et al. 1997; Wegmann et al. 1998; Schwadron \& Cravens 2000; Kharchenko et al. 2003; Otranto et al. 2007) and has been the subject of numerous reviews (Cravens 2002; Krasnopolskv et al. 2004; Bhardwaj et al. 2007; Dennerl 2008). It has been argued that cometary $\mathrm{X}$-rays represent a potential tool to monitor not only cometary activity, but also the composition, velocity, and flux of the SW in regions that spacecraft cannot reach (Cravens 1997; 
Dennerl et al. 1997; Schwadron \& Cravens 2000; Beiersdorfer et al. 2001).

It is now also recognized that heliospheric Xray emission due to SWCX with $\mathrm{H}$ and $\mathrm{He}$ interstellar neutrals (see, e.g., Cox 1998; Cravens 2000; Pepino et al. 2004; Robertson et al. 2009, and references therein), and X-ray generation throughout the terrestrial magnetosheath due to SWCX with geocoronal neutrals (see, e.g., Dennerl et al. 1997; Cox 1998; Cravens et al. 2009, and references therein) contribute to the soft X-ray background (SXRB). SWCX with $\mathrm{H}$ and $\mathrm{O}$ has also been proposed to account for the first definite detection of X-ray emission from the exosphere of Mars (Dennerl et al. 2006).

Understanding and accurately predicting these and related phenomena require novel experiments which simultaneously measure detailed (i.e., charge and quantum state-resolved) collision parameters in coincidence with consequent atomic energy de-excitation to elucidate the underlying chain of mechanisms in this CX induced X-ray emission, and ultimately, development of theoretical methods capable of broadly treating such interactions. To this end, several experimental groups have carried out laboratory studies of relevant collision systems (see, e.g., Beiersdorfer et al. 2000, 2001, 2003; Greenwood et al. 2001; Hasan et al. 2001; Gao \& Kwong 2004; Ali et al. 2005; Bodewits et al. 2006; Mawhorter et al. 2007; Allen et al. 2008; Diurić et al. 2008, and references therein). Of particular importance for accurate modeling is the ability to predict the $n \ell$-state-selective CX cross sections (i.e., to account for the distributions of the principal $n$ and angular momentum $\ell$ quantum numbers of the product projectile ions). All previous modeling attempts to simulate cometary or heliospheric X-ray spectra (Häberli et al. 1997; Wegmann et al. 1998; Rigazio et al. 2002; Beiersdorfer et al.|2003; Kharchenko et 2003; Otranto et al. 2007; Otranto \& Olson 2008) have adopted simple $n \ell$ empirical relations, scalings from related collision systems, or fits to laboratory non-coincident total X-ray spectra. It should be noted, however, that non-coincident laboratory spectra contain contributions from a variety of reaction channels such as single electron capture (SEC) and autoionizing and nonautoionizing multiple-electron capture (MEC). A superposition of several reaction channels is also likely to occur in cometary, planetary, and heliospheric spectra. Therefore, a technique which is capable of differentiating between the primary reaction channels is required for the interpretation of such spectra.

In this letter, we report an experimental investigation of the $n$-state-selective hydrogen-like ion X-ray spectra following SEC in collisions of $\mathrm{Ne}^{10+}$ with $\mathrm{He}$, Ne, and Ar neutral targets at a laboratory frame collision velocity $v$ of $933 \mathrm{~km}$ $\mathrm{s}^{-1}\left(4.54 \mathrm{keV}^{\text {nucleon }}{ }^{-1}\right)$. This velocity is at the upper end of the SW ion velocities. The present interactions are close analogs of the interactions of heavy minor, multiply charged, SW ions with cometary, planetary, and heliospheric neutrals. Specifically, the dominantly molecular constituents of cometary and planetary atmospheres (e.g., $\mathrm{H}_{2} \mathrm{O}, \mathrm{CO}_{2}$ ) are simulated by gases of similar ionization potential and multielectron character (i.e., Ar, Ne). Helium, being the second most abundant (15\%) interstellar neutral (Koutroumpa et al. 2009), is of direct relevance to heliospheric X-ray emission. The present spectra are free from complications arising from the inability of previously employed non-coincident total $\mathrm{X}$-ray spectra to differentiate between the primary reaction channels. Consequently they offer the opportunity to test critically the ability of theories to describe SWCX interactions at the $n \ell$ quantum level.

\section{Experiment}

Simultaneous cold-target recoil ion momentum spectroscopy (COLTRIMS) and X-ray spectroscopy were used for the triple-coincident detection of X-rays, scattered projectiles, and target recoil ions. COLTRIMS has been reviewed by Dörner et al. (2000), while the components of the experimental apparatus have been described elsewhere (Hasan et al. 1999; Ali et al. 2005). Briefly, the ${ }^{22} \mathrm{Ne}^{10+}$ ions were provided by the University of Nevada, Reno, $14 \mathrm{GHz}$ ECR ion source, and guided to the collision chamber where they crossed supersonic target jets at $90^{\circ}$. The target recoil ions resulting from the collisions were extracted by an electric field, at $90^{\circ}$ relative to the incident ions and jet, and detected by a positionsensitive detector (PSD). The scattered projectile 
ions were charge analyzed electrostatically and detected by another PSD where their impact positions provided their final charge states, while coincident time-of-flight (TOF) measurements between projectile and recoil ions provided the recoil ion charge states. X-rays emitted at $90^{\circ}$ relative to the incident ions were detected by a windowless high-purity germanium detector, placed opposite the recoil detector. Coincidences between projectile ions and X-rays ensured that all detected particles originated in the same collision event. The full-width-at-half-maximum (FWHM) of the $\mathrm{X}$-ray peaks is energy-dependent with a value of about $126 \mathrm{eV}$ for the $\mathrm{Ne}^{9+} \mathrm{Ly} \alpha$ line $(1021.8 \mathrm{eV})$ and $\sim 133 \mathrm{eV}$ for the $\mathrm{Ly} \delta$ line $(1307.7 \mathrm{eV})$.

\section{Results, theory, and discussion}

Since no electrons are directly ejected to the continuum for the considered collision energy, the change in electronic energy of the collision system, or the $Q$-value, is a direct measure of the projectile state population immediately following the collision. $Q$-value spectra, therefore, provide the experimental $n$-state-selective relative cross sections $\sigma_{n}^{\text {rel }}$. The $Q$-value for SEC is given by $Q \approx-\left(P_{\|} v+v^{2} / 2\right)$ (Ali et al. 1992), where $P_{\|}$is the longitudinal (i.e., parallel to the incident projectile direction) momentum transfer to the recoiling target. $P_{\|}$of the recoil ions were determined from their TOF and impact positions on the PSD. Figure 1 displays the $Q$-value spectra for pure SEC for the three considered collision systems with the $\sigma_{n}^{\text {rel }}$ indicated. These spectra were obtained using COLTRIMS-only measurements to acquire sufficient statistics for the accurate determination of $\sigma_{n}^{\text {rel }}$.

Non-perturbative quantum mechanical treatments of highly charged ion collisions with multielectron targets are difficult. A recent study of $\mathrm{Cl}^{7+}$ collisions with $\mathrm{H}$ (Zhao et al. 2007) demonstrates that such calculations are not routine, but require particular care, significant computational resources, and individual consideration. Therefore, we adopt a more tractable approach to model the measured interactions: the well-established classical trajectory Monte-Carlo (CTMC) method (Olson \& Salop 1977). The basic CTMC approach for bare ion collisions involving single-valence electron targets was discussed in Hasan et al. (2001), while elaborations for multielectron targets in the context of total MEC was given in Ali et al. (2005). The He target was treated using the former method with the electron-nuclear charge interaction described by an effective charge $Z_{\text {eff }}=$ 1.6875, designated as nCTMC (i.e., "CTMC" for "n" electrons bound by the atom's sequential ionization potentials). For the Ne and Ar targets, six valence electrons within the independent particle model were considered (inCTMC). The electronatomic core interaction was treated with a model potential. The standard microcanonical ensemble for the initial electron orbitals was filtered to remove all but the $p$ orbitals.

Examination of the experimental and CTMC $\sigma_{n}^{\text {rel }}$ displayed in Fig. 1 clearly shows that the agreement is excellent for both the He and Ne targets and reasonable for Ar. Reasonable agreement between experiment and CTMC has been reported previously by Cassimi et al. (1996) for $\mathrm{Ne}^{10+}$ and $\mathrm{Ar}^{18+}$ on $\mathrm{He}$ at a slightly higher collision velocity $\left(\approx 1143 \mathrm{~km} \mathrm{~s}^{-1}\right)$. That reasonable success has prompted the use of CTMC $\sigma_{n \ell}$ to simulate non-coincident total cometary and laboratory Xray spectra (Otranto et al. 2007; Otranto \& Olson 2008) under the assumption that the spectra are dominated by SEC. As we previously demonstrated (Ali et al. 2005), these spectra may contain significant contributions from MEC. Furthermore, very high resolution $(\mathrm{FWHM} \approx 10 \mathrm{eV})$ total X-ray spectra using an X-ray microcalorimeter show clear signatures of MEC (Beiersdorfer et al. 2003). These and similar signatures in later work could not be accounted for by other SEC CTMC calculations (Otranto et al. 2007), and the relative contributions to certain high energy emission lines from SEC and MEC remain in question (Wargelin et al. 2005, 2008). Total X-ray spectra are, therefore, not the appropriate benchmarks for testing the validity of theoretical CX methods for simulating X-ray spectra.

A major advantage of the simultaneous COLTRIMS and X-ray spectroscopic measurements is that it is not only possible to separate X-rays originating in pure SEC from those due to MEC (Ali et al. 2005), but it is also possible to obtain X-ray spectra corresponding to each populated $n$-level in the pure SEC channel as given in Figs. 2,4. The present measurements also provide the opportunity to test the validity of the theoretical methods, 


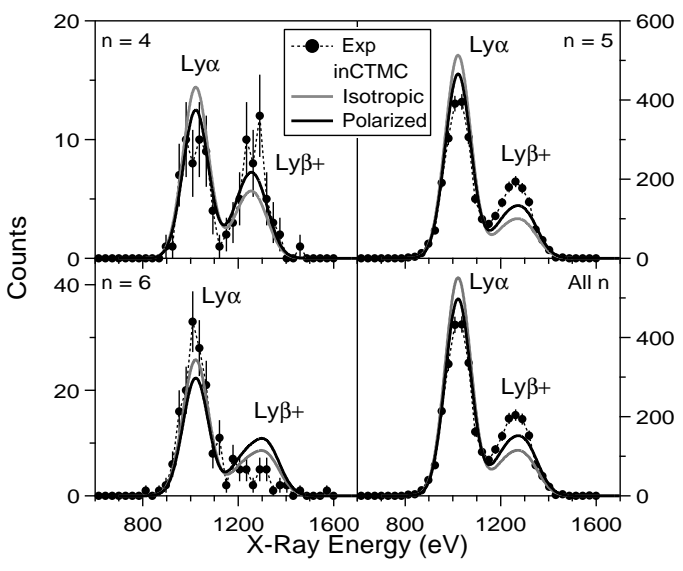

Fig. 3.- Same as in Fig. 2 but for SEC from Ne.

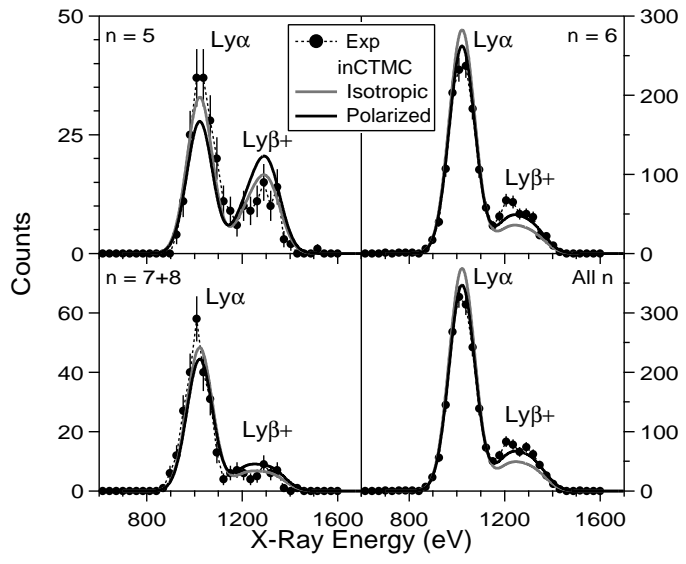

Fig. 4.- Same as in Fig. 2 but for SEC from Ar. be well founded. This complements results from earlier investigations which showed that the dominance of SEC assumed in the models is also not justified (Ali et al. 2005).

While the most recent X-ray emission simulations represent a major improvement over earlier efforts, which adopted equipartition or statistical angular momentum models (Häberli et al. 1997; Wegmann et al. 1998; Schwadron \& Cravens 2000), the present results suggest that agreement with observations is likely to be fortuitous. Deductions of relevant parameters from such models should be considered as being associated with appreciable systematic uncertainties.

Considering the success of CTMC in accounting for $\sigma_{n}^{\text {rel }}$ as shown in Fig. 1, it may seem surprising that it underestimates the $\mathrm{Ly} \beta+$ intensity for the dominant $n$-levels. CTMC, however, has a propensity towards a more statistical $\ell$-distribution (i.e., $\left.\sigma_{\ell} \propto(2 \ell+1)\right)$, at least for the lower $\ell$ 's. Further, the agreement is seen to improve with decreasing first ionization potential: $\mathrm{He}(24.6 \mathrm{eV}), \mathrm{Ne}(21.6 \mathrm{eV})$, and $\mathrm{Ar}(15.8 \mathrm{eV})$. In fact, the best agreement occurs for Ar which might be taken as a surrogate for the dominant species in cometary and planetary atmospheres responsible for the X-ray emission: $\mathrm{H}_{2}(15.4 \mathrm{eV}), \mathrm{CO}$ $(14.0 \mathrm{eV}), \mathrm{CO}_{2}(13.7 \mathrm{eV})$, and $\mathrm{H}_{2} \mathrm{O}(12.6 \mathrm{eV})$. The observation that the largest discrepancies occur for He suggests that the problem lies in electroncorrelation effects which are known to be strongest for the He atom. Electron correlation is not sufficiently accounted for in the present CTMC calculations.

\section{Conclusions}

In summary, relative state-selective cross sections and X-ray spectra have been obtained from triple-coincident measurements of X-rays, scattered projectiles, and target recoil ions which provide a test of CX theories at the quantum orbital angular momentum level. While improvements in $\mathrm{X}$-ray simulations based on CX models have been made, the current results show that agreement for the relative state-selective cross sections does not necessarily imply agreement for the state selective X-ray spectra, and suggest that comparison of such models to observations of solar system Xrays may lead to faulty conclusions or parameter 
extractions.

Support is acknowledged from US DOE contract No. DE-AC52-07NA-27344 and NASA grant NNG06GB11G (PB), NASA grant NNH07AF12I (DRS), and NASA grants NNG05GD98G and NNX09AC46G (PCS). RA acknowledges partial support from NASA EPSCoR, Nevada Astrophysics Program. RA, PB, DRS, and PCS thank the NSF-funded Institute for Theoretical Atomic, Molecular, and Optical Physics at the HarvardSmithsonian Center for Astrophysics for travel support.

\section{REFERENCES}

Ali, R., Frohne, V., Cocke, C. L., Stöckli, M., Cheng, S., \& Raphaelian, M. L. A. 1992, Phys. Rev. Lett., 69, 2491

Ali, R., et al. 2005, ApJ, 629, L125

Allen, F. I., Biedermann, C., Radtke, R., \& Fussmann, G. 2008, Phys. Rev. A, 78, 032705

Beiersdorfer, P., et al. 2000, Phys. Rev. Lett., 85, 5090

Beiersdorfer, P., Lisse, C. M., Olson, R. E., Brown, G. V., \& Chen, H. 2001, ApJ, 549, L147

Beiersdorfer, P., et al. 2003, Science, 300, 1558

Bhardwaj, A., et al. 2007, Planet. Space Sci., 55, 1135

Bodewits, D., Hoekstra, R., Seredyuk, B., McCullough, R. W., Jones, G. H., \& Tielens, A. G. G. M. 2006, ApJ, 642, 593

Bodewits, D., et al. 2007,A\&A, 469, 1183

Cassimi, A., Duponchel, S., Flechard, X., Jardin, P., Sortais, P., Hennecart, D., \& Olson, R. E. 1996, Phys. Rev. Lett.76, 3679

Cravens, T. E. 1997, Geophys. Res. Lett., 24, 105

Cravens, T. E. 2000, ApJ, 532, L153

Cravens, T. E. 2002, Science, 296, 1042

Cravens, T. E., Robertson, I. P., Snowden S., Kuntz, K., Collier, M., \& Medvedev, M. 2009, in AIP Conf. Proc. 1156, Local Bubble and Beyond II, ed. Kuntz, K. D., Smith, R. K., \& Snowden, S. L. (AIP, New York), 37
Cox, D. P. 1998, in Lect. Notes Phys. 506, The Local Bubble and Beyond, ed. Breitschwerdt, D., Freyberg, M. J., \& Trümper, J. (Springer, Berlin), 121

Dennerl, K., Englhauser, J., \& Trümper, J. 1997, Science, 277, 1625

Dennerl, K., et al. 2006, A\&A, 451, 709

Dennerl, K. 2008, in The Universe in X-Rays, ed. Trümper, J. E. \& Hasings G. (Springer-Verlag Berlin Heidelberg), 85

Djurić, N., Smith, S. J., Simcic, J., \& Chutjian, A. 2008, ApJ, 679, 1661

Dörner, R., Mergel, V., Jagutzki, O., Spielberger, L., Ullrich, J., Moshammer, R., \& SchmidtBocking, H. 2000, Phys. Reports, 330, 95

Gao, H., \& Kwong, V. H. S. 2004, Phys. Rev. A, 69,052715

Greenwood, J. B., Williams, I. D., Smith, S. J., \& Chutjian, A. 2001, Phys. Rev. A, 63, 062707

Häberli, R. M., Gombosi, T. I., DeZeeuw, D. L., Combi, M. R., \& Powell, K. G. 1997, Science, 276,939

Hasan, A. A., Emmons, E. D., Hinojosa, G., \& Ali, R. 1999, Phys. Rev. Lett., 83, 4522

Hasan, A. A., Eissa, F., Ali, R., Schultz, D. R., \& Stancil, P. C. 2001, ApJ, 560, L205

Kharchenko, V., Rigazio, M., Dalgarno, A., \& Krasnopolsky, V. A. 2003, ApJ, 585, L73

Koutroumpa, D., Collier, M. R., Kuntz, K. D., Lallement, R., \& Snowden, S. L. 2009, ApJ, 697,1214

Krasnopolsky, V. A., \& Mumma, M. J. 2001, ApJ, 549, 629

Krasnopolsky, V. A., Christian, D. J., Kharchenko, V., Dalgarno, A., Wolk, S. J., Lisse, C. M., \& Stern, S. A. 2002, Icarus, 160,437

Krasnopolsky, V. A., Greenwood, J. B., \& Stancil, P. C. 2004, Space Sci. Rev., 113, 271

Lisse, C. M., et al. 1996, Science, 274, 205 
Lisse, C. M., Christian, D. J., Dennerl, K., Meech, K. J., Petre, R., Weaver, H. A., \& Wolk, S. J. 2001, Science, 292, 1343

Lisse, C. M., et al. 2007, Icarus, 190, 391

Mawhorter, R. J., et al. 2007, Phys. Rev. A, 75, 032704

Olson, R. E., \& Salop, A. 1977, Phys. Rev. A, 15, 531

Otranto, S., Olson, R. E., \& Beiersdorfer, P. 2007, J. Phys. B, 40, 1755

Otranto, S., \& Olson, R. E. 2008, Phys. Rev. A, 77,022709

Pepino, R., Kharchenko, V., Dalgarno, A., \& Lallement, R. 2004, ApJ, 617, 1347

Rigazio, M., Kharchenko, V., \& Dalgarno A. 2002, Phys. Rev. A, 66, 064701

Robertson, I. P., Kuntz, K. D., Collier, M. R., Cravens, Thomas E., \& Snowden, S. L. 2009, in AIP Conf. Proc. 1156, Local Bubble and Beyond II, ed. Kuntz, K. D., Smith, R. K., \& Snowden, S. L. (AIP, New York), 52

Schwadron, N. A., \& Cravens, T. E. 2000, ApJ, 544,558

Vernhet, D., et al. 1985, Phys. Rev. A, 32, 1256

Wargelin, B. J., Beiersdorfer, P., Scofield, J. H., Neill, P. A., \& Olson, R. E. 2005, ApJ, 634, 687

Wargelin, B. J., Beiersdorfer, P., \& Brown, G. V. 2008, Canadian J. of Phys., 86, 161

Wegmann, R., Schmidt, H. U., Lisse, C. M., Dennerl, K., \& Englhauser, J. 1998, Planet. Space Sci., 46, 603

Willingale, R., et al. 2006, ApJ, 649, 541

Zhao, L. B., Watanabe, A., Stancil, P. C., \& Kimura, M. 2008, Phys. Rev. A, 76, 022701

This 2-column preprint was prepared with the AAS LATEX macros v5.2. 